\title{
Parameterized GSOR Method for a Class of Complex Symmetric Systems of Linear Equations
}

\author{
Yu-Jiang $\mathrm{Wu}^{1, *}$, Wei-Hong Zhang ${ }^{2}, \mathrm{Xi}^{-} \mathrm{An} \mathrm{Li}^{3}$ and Ai-Li Yang ${ }^{1}$ \\ ${ }^{1}$ School of Mathematics and Statistics/Gansu Key Laboratory of Applied \\ Mathematics and Complex Systems, Lanzhou University, Lanzhou 730000, P.R. Chi- \\ na \\ 2 School of Mathematics and Statistics, Lanzhou University, Lanzhou 730000, P.R. \\ China \\ 3 School of Mathematical Sciences, Shanghai Jiao Tong University, Shanghai 200240, \\ P.R. China.
}

Received January 23, 2018; Accepted February 28, 2018

\begin{abstract}
A parameterized generalized successive overrelaxation (PGSOR) method for a class of block two-by-two linear system is established in this paper. The convergence theorem of the method is proved under suitable assumptions on iteration parameters. Besides, we obtain a functional equation between the parameters and the eigenvalues of the iteration matrix for this method. Furthermore, an accelerated variant of the PGSOR (APGSOR) method is also presented in order to raise the convergence rate. Finally, numerical experiments are carried out to confirm the theoretical analysis as well as the feasibility and the efficiency of the PGSOR method and its variant.
\end{abstract}

AMS subject classifications: 65F10, 65F50, 65F08

Key words: Complex linear systems, symmetric positive definite, spectral radius, convergence, preconditioning.

\section{Introduction}

Consider the system of linear equations

$$
A u=b, \quad A \in \mathbb{C}^{n \times n}, u, b \in \mathbb{C}^{n},
$$

where $A$ is a complex symmetric matrix with the form

$$
A=W+i T, \quad(i=\sqrt{-1}),
$$

*Corresponding author. Email addresses: myjaw@lzu.edu.cn (Y.-J. Wu), zhangwh13@lzu.edu.cn (W.-H. Zhang), lixian9131@163.com (X.-A. Li), yangaili@lzu.edu.cn (A.-L. Yang) 
and $W, T \in \mathbb{R}^{n \times n}$ are both symmetric matrices with at least one of them being positive definite. Hereafter, without loss of generality, we assume that $W$ is symmetric positive definite.

It is known to all that the Equation (1.1) arises frequently in many scientific and engineering applications. For instance, it comes from diffuse optimal tomography [1], molecular scattering [2], wave propagation [3], structural dynamics [4], FFT-based solution of certain time-dependent PDEs [5] and so on. More examples and additional practical backgrounds can be found in other places. See, e.g., [6] and references therein.

During these years, many methods with iteration approaches have been proposed for significantly approximating the unique solution of this system (1.1). For example, some of the well-known preconditioned Krylov subspace methods $[5,7,8]$, Hermitian and skew-Hermitian splitting (HSS) method and lots of its variants [9-15], and C-to-R iteration methods [16-19] are proven to be useful techniques for solving the symmetric linear systems. As a matter of fact, our system (1.1) can be easily changed into a special case of generalized saddle point problems, so several generalizations of classical methods, such as generalized successive overrelaxation (GSOR) method in [20], the new variations of the method in [21-23] have brought new insight and new tools for solving such systems.

Based on GSOR method and some of its generalization, we in this paper develop a parameterized generalized SOR (PGSOR) method for solving the complex symmetric linear system (1.1). We study also the convergence properties and some its variants.

The organization of the paper is as follows. In Section 2, the new method PGSOR is established. In Section 3, the convergence analysis of the PGSOR method is exactly considered based on some lemmas. Section 4 will construct an efficient preconditioner to accelerate the convergence of the PGSOR method. In Section 5, some numerical examples are presented to show good behaviors for the efficiency of our methods. Finally, the paper is concluded in Section 6.

\section{Parameterized GSOR method}

Let $u=x+i y$ and $b=p+i q$, where $x, y, p, q \in \mathbb{R}^{n}$. Then the system (1.1) becomes

$$
(W+i T)(x+i y)=p+i q .
$$

And the complex linear system can be equivalently written as a block two-by-two real equivalent formulation

$$
\mathcal{A} \widetilde{u}:=\left(\begin{array}{cc}
W & -T \\
T & W
\end{array}\right)\left(\begin{array}{l}
x \\
y
\end{array}\right)=\left(\begin{array}{c}
p \\
q
\end{array}\right)=: \widetilde{b} .
$$

Inasmuch $W$ is symmetric positive definite and $T$ is symmetric, then the coefficient matrix $\mathcal{A}$ is nonsingular. A natural splitting of $\mathcal{A}$ is as follow,

$$
\mathcal{A}=\mathcal{D}-\mathcal{L}-\mathcal{U},
$$


where

$$
\mathcal{D}=\left(\begin{array}{cc}
W+\tau I & 0 \\
0 & W
\end{array}\right), \quad \mathcal{L}=\left(\begin{array}{cc}
0 & 0 \\
-T & 0
\end{array}\right), \quad \mathcal{U}=\left(\begin{array}{cc}
\tau I & T \\
0 & 0
\end{array}\right)
$$

and $\tau$ is a nonnegative real parameter, $I_{n} \in \mathbb{R}^{n \times n}$ is the n-by-n identity matrix.

Our PGSOR method is considered to be an iteration scheme for solving the linear system (2.2), i.e.,

$$
\left(\begin{array}{c}
x^{(k+1)} \\
y^{(k+1)}
\end{array}\right)=(\mathcal{D}-\alpha \mathcal{L})^{-1}[(1-\alpha) \mathcal{D}+\alpha \mathcal{U}]\left(\begin{array}{c}
x^{(k)} \\
y^{(k)}
\end{array}\right)+\alpha(\mathcal{D}-\alpha \mathcal{L})^{-1}\left(\begin{array}{c}
p \\
q
\end{array}\right) .
$$

It is equivalent to

$$
\left(\begin{array}{c}
x^{(k+1)} \\
y^{(k+1)}
\end{array}\right)=\mathcal{H}_{(\alpha, \tau)}\left(\begin{array}{c}
x^{(k)} \\
y^{(k)}
\end{array}\right)+\mathcal{G}_{(\alpha, \tau)}^{-1}\left(\begin{array}{c}
p \\
q
\end{array}\right)
$$

where

$$
\mathcal{H}_{(\alpha, \tau)}=\left(\begin{array}{ll}
H_{11} & H_{12} \\
H_{21} & H_{22}
\end{array}\right)
$$

with

$$
\left\{\begin{array}{l}
H_{11}=(1-\alpha)(W+\tau I)^{-1} W+\tau(W+\tau I)^{-1}, \\
H_{12}=\alpha(W+\tau I)^{-1} T, \\
H_{21}=-\alpha(1-\alpha) W^{-1} T(W+\tau I)^{-1} W-\alpha \tau W^{-1} T(W+\tau I)^{-1}, \\
H_{22}=(1-\alpha) I-\alpha^{2} W^{-1} T(W+\tau I)^{-1} T,
\end{array}\right.
$$

and

$$
\mathcal{G}_{(\alpha, \tau)}=\left(\begin{array}{cc}
\frac{1}{\alpha} W+\frac{\tau}{\alpha} I & 0 \\
T & \frac{1}{\alpha} W
\end{array}\right)
$$

Now the algorithm for the PGSOR method can be described as follows.

\section{Algorithm 2.1. The PGSOR method}

Given initial vectors $x^{(0)} \in \mathbb{R}^{n}$ and $y^{(0)} \in \mathbb{R}^{n}$, and two real relaxation factors $\alpha>0, \tau \geq 0$. For $k=0,1,2, \ldots$ until the iteration sequence $\left\{\left(x^{(k)^{T}}, y^{(k)^{T}}\right)^{T}\right\}$ converges to the exact solution, compute the next iteration according to the following procedure

$$
\left\{\begin{array}{l}
(W+\tau I) x^{(k+1)}=(1-\alpha) W x^{(k)}+\tau x^{(k)}+\alpha T y^{(k)}+\alpha p \\
W y^{(k+1)}=(1-\alpha) W y^{(k)}-\alpha T x^{(k+1)}+\alpha q .
\end{array}\right.
$$

Meantime, it is not hard to find that the PGSOR method will reduce to the GSOR method when $\tau=0$. 


\section{Convergence analysis for the PGSOR method}

For convergence analysis we need the following lemmas.

Lemma 3.1. If $\lambda$ is an eigenvalue of matrix $\mathcal{H}_{(\alpha, \tau)}$, then $\lambda \neq 1$.

Proof. Suppose $\lambda$ is the eigenvalue of $\mathcal{H}_{(\alpha, \tau)}$, and $X=\left(x^{T}, y^{T}\right)^{T}$ is the corresponding eigenvector. Then $\mathcal{H}_{(\alpha, \tau)} X=\lambda X$, that is

$$
(\mathcal{D}-\alpha \mathcal{L})^{-1}[(I-\alpha) \mathcal{D}+\alpha \mathcal{U}] X=\lambda X,
$$

or equivalently,

$$
\left(\begin{array}{cc}
(1-\alpha) W+\tau I & \alpha T \\
0 & (1-\alpha) W
\end{array}\right)\left(\begin{array}{l}
x \\
y
\end{array}\right)=\lambda\left(\begin{array}{cc}
W+\tau I & 0 \\
\alpha T & W
\end{array}\right)\left(\begin{array}{l}
x \\
y
\end{array}\right) .
$$

Evidently we have

$$
\left\{\begin{array}{l}
{[(1-\alpha) W+\tau I] x+\alpha T y=\lambda(W+\tau I) x,} \\
(1-\alpha) W y=\lambda \alpha T x+\lambda W y .
\end{array}\right.
$$

If $\lambda=1$, then

$$
T y=W x, \quad T x=-W y .
$$

That means exactly $\left(T W^{-1} T+W\right) y=0$. Because $W$ is a symmetric positive definite matrix and $T$ is a symmetric matrix, $T W^{-1} T+W$ is nonsingular. This implies that $y=0$, thereby $x=0$. It shows that the corresponding eigenvector of $\lambda$ is a zero vector. This contradiction leads to the conclusion: $\lambda \neq 1$.

Lemma 3.2. ([20]) Let the matrices $W$ and $T \in \mathbb{R}^{n \times n}$ be symmetric positive definite and symmetric, respectively. Then the eigenvalues of the matrix $S=W^{-1}$ T are all real.

Lemma 3.3. ([24]) Both roots of real quadratic equation $x^{2}-b x+c=0$ are less than one in modulus if and only if $|c|<1$ and $|b|<1+c$.

Applying the above Lemmas, we are able to have the conclusion about the eigenvalues of the iteration matrix of the PGSOR method which will play an important role in the proof process for convergence.

Theorem 3.1. Let $W$ and $T \in \mathbb{R}^{n \times n}$ be symmetric positive definite and symmetric, respectively. And let $\tau$ be a nonnegative real constant. Assume that $\lambda$ is an eigenvalue of the iteration matrix $\mathcal{H}_{(\alpha, \tau)}$ and $X=\left(x^{T}, y^{T}\right)^{T} \in \mathbb{R}^{2 n}$ is the corresponding eigenvector. Denote by

$$
\eta=\frac{x^{T} S x}{x^{T} x}, \mu=\frac{x^{T} W^{-1} x}{x^{T} x},
$$

where $S=W^{-1} T$. Then either $\lambda=1-\alpha$ or $\lambda$ satisfies the quadratic equation

$$
\lambda^{2}-\lambda \frac{2(1-\alpha)+\tau \mu(2-\alpha)-\alpha^{2} \eta^{2}}{1+\tau \mu}+\frac{(1-\alpha)(1-\alpha+\tau \mu)}{1+\tau \mu}=0 .
$$


Proof. From Lemma 3.1, we have known that $\mathcal{H}_{(\alpha, \tau)} X=\lambda X$. Thus

$$
\left(\begin{array}{cc}
(1-\alpha) W+\tau I & \alpha T \\
0 & (1-\alpha) W
\end{array}\right)\left(\begin{array}{l}
x \\
y
\end{array}\right)=\lambda\left(\begin{array}{cc}
W+\tau I & 0 \\
\alpha T & W
\end{array}\right)\left(\begin{array}{l}
x \\
y
\end{array}\right) .
$$

So, we have the following system of two equations

$$
\left\{\begin{array}{l}
{[(1-\alpha) W+\tau I] x+\alpha T y=\lambda(W+\tau I) x,} \\
(1-\alpha) W y=\lambda \alpha T x+\lambda W y .
\end{array}\right.
$$

In the system we can assert that $y \neq 0$. Otherwise, if $y=0$, it is not difficult to find that $x=0$. This is contradiction. Recalling that $\lambda \neq 1$, so (3.3) changes directly into the following system,

$$
\left\{\begin{array}{l}
(1-\alpha-\lambda) x+\tau(1-\lambda) W^{-1} x+\alpha W^{-1} \mathrm{~T} y=0, \\
(1-\alpha-\lambda) y-\lambda \alpha W^{-1} \mathrm{~T} x=0 .
\end{array}\right.
$$

If $\lambda=1-\alpha$ and $W^{-1} T$ is a singular matrix, the equation (3.3) reduces to

$$
\left\{\begin{array}{l}
\tau W^{-1} x+W^{-1} T y=0 \\
\left(\alpha^{2}-\alpha\right) W^{-1} T x=0
\end{array}\right.
$$

So we get

$$
x \in \operatorname{null}\left(W^{-1} T\right) \text { and } \tau W^{-1} x+W^{-1} T y=0 .
$$

If $\lambda=1-\alpha$ and $W^{-1} T$ is a nonsingular matrix, it is not hard to verify that both $x=0$ and $y=0$. The result will contradict that $y \neq 0$.

If $\lambda \neq 1-\alpha$, from the second equality in (3.4), we have

$$
y=\frac{\lambda \alpha}{1-\alpha-\lambda} W^{-1} T x
$$

Substituting it into the first equality in (3.4), one obtains

$$
(1-\alpha-\lambda) x+\tau(1-\lambda) W^{-1} x+\frac{\lambda \alpha^{2}}{(1-\alpha-\lambda)} W^{-1} T W^{-1} T x=0 .
$$

If we let $S=W^{-1} T$ and denote by $\eta=\left(x^{T} S x\right) /\left(x^{T} x\right), \mu=\left(x^{T} W^{-1} x\right) /\left(x^{T} x\right)$, then

$$
(1-\alpha-\lambda)+\tau \mu(1-\lambda)+\frac{\lambda \alpha^{2} \eta^{2}}{(1-\alpha-\lambda)}=0
$$

Hence comes,

$$
\lambda^{2}-\lambda \frac{2(1-\alpha)+\tau \mu(2-\alpha)-\alpha^{2} \eta^{2}}{1+\tau \mu}+\frac{(1-\alpha)(1-\alpha+\tau \mu)}{1+\tau \mu}=0 .
$$

This completes the proof of the theorem. 
Theorem 3.2. Let $W$ and $T \in \mathbb{R}^{n \times n}$ be symmetric positive definite and symmetric, respectively. Also let $\tau$ be a nonnegative real constant and $\mu$ be the quantity defined in Theorem 3.1. Then the PGSOR method is convergent if and only if $\alpha$ satisfies

$$
0<\alpha<2
$$

or

$$
\left\{\begin{array}{l}
0<\alpha<\min \left\{2+\tau \mu_{\min }, \frac{2+\tau \mu_{\min }-\sqrt{\left(2+\tau \mu_{\min }\right)^{2}-4\left(1+\tau \mu_{\min }\right)\left(1-\rho^{2}(S)\right)}}{1-\rho^{2}(S)}\right\}, \\
0<\alpha<\frac{2+2 \tau \mu_{\min }}{2+\tau \mu_{\min }}, \text { if } 1-\rho^{2}(S)=0,
\end{array}\right.
$$

where $\rho(S)$ is the spectral radius of the matrix $S=W^{-1} T$.

Proof. By Theorem 3.1 we know that, if $\lambda=1-\alpha$, then $|\lambda|<1$ is equivalent to $0<\alpha<2$.

While if $\lambda$ satisfies the quadratic Equation (3.2), then the Lemma 3.3 applies. It tells us that $|\lambda|<1$ is equivalent to

$$
\begin{aligned}
& \left|\frac{(1-\alpha)(1-\alpha+\tau \mu)}{1+\tau \mu}\right|<1, \\
& \left|\frac{2(1-\alpha)+\tau \mu(2-\alpha)-\alpha^{2} \eta^{2}}{1+\tau \mu}\right|<1+\frac{(1-\alpha)(1-\alpha+\tau \mu)}{1+\tau \mu} .
\end{aligned}
$$

Finding the solution of the first inequality in (3.5) and noticing that

$$
0<\alpha<2+\tau \mu_{\min }<2+\tau \mu,
$$

immediately we can obtain that

$$
\left|\frac{(1-\alpha)(1-\alpha+\tau \mu)}{1+\tau \mu}\right|<1
$$

As for the second inequality in (3.5), we have

$$
\left\{\begin{array}{l}
-(1-\alpha)(1-\alpha+\tau \mu)-1-\tau \mu<2(1-\alpha)+\tau \mu(2-\alpha)-\alpha^{2} \eta^{2} \\
2(1-\alpha)+\tau \mu(2-\alpha)-\alpha^{2} \eta^{2}<1+\tau \mu+(1-\alpha)(1-\alpha+\tau \mu) .
\end{array}\right.
$$

It is easily to see that the first inequality in (3.6) naturally hold for any $\alpha>0$. Now let us consider the second inequality. By calculating, we have

$$
\alpha^{2}-2(2+\tau \mu) \alpha+4(1+\tau \mu)>\alpha^{2} \eta^{2} .
$$

According to Theorem 3.1, we can observe from the previous inequality

$$
\alpha^{2}-2\left(2+\tau \mu_{\min }\right) \alpha+4\left(1+\tau \mu_{\min }\right)>\alpha^{2} \rho^{2}(S),
$$


which leads to

$$
\left(1-\rho^{2}(S)\right) \alpha^{2}-2\left(2+\tau \mu_{\min }\right) \alpha+4\left(1+\tau \mu_{\min }\right)>0 .
$$

Combining $0<\alpha<2+\tau \mu_{\min }$, direct computation gives that if $1-\rho^{2}(S) \neq 0$, then

$$
0<\alpha<\min \left\{2+\tau \mu_{\min }, \frac{2+\tau \mu_{\min }-\sqrt{\left(2+\tau \mu_{\min }\right)^{2}-4\left(1+\tau \mu_{\min }\right)\left(1-\rho^{2}(S)\right)}}{1-\rho^{2}(S)}\right\},
$$

else

$$
0<\alpha<\frac{2+2 \tau \mu_{\min }}{2+\tau \mu_{\min }} .
$$

In conclusion, we know that the result of this theorem holds. The proof is completed.

\section{Accelerating the PGSOR method}

Additionally, if we assume that the matrices $W$ and $T$ are symmetric positive definite and symmetric positive semi-definite respectively, then a preconditioner to accelerate the convergence rate of the PGSOR method can be introduced. In fact, let $I \in \mathbb{R}^{n \times n}$ be an identity, and we consider the following preconditioned form of (2.2)

$$
\left(\begin{array}{cc}
I & I \\
-I & I
\end{array}\right)\left(\begin{array}{cc}
W & -T \\
T & W
\end{array}\right)\left(\begin{array}{l}
x \\
y
\end{array}\right)=\left(\begin{array}{cc}
I & I \\
-I & I
\end{array}\right)\left(\begin{array}{l}
p \\
q
\end{array}\right)
$$

or equivalently,

$$
\left(\begin{array}{cc}
\widetilde{W} & -\widetilde{T} \\
\widetilde{T} & \widetilde{W}
\end{array}\right)\left(\begin{array}{l}
x \\
y
\end{array}\right)=\left(\begin{array}{c}
\widetilde{p} \\
\widetilde{q}
\end{array}\right)
$$

where $\widetilde{W}:=W+T, \widetilde{T}:=T-W$ and $\widetilde{p}:=p+q, \widetilde{q}:=q-p$. Applying the PGSOR method to the linear system (4.1), we have the APGSOR method for the linear system (2.2).

Algorithm 4.1. The APGSOR method

Given initial vectors $x^{(0)} \in \mathbb{R}^{n}$ and $y^{(0)} \in \mathbb{R}^{n}$, and two real relaxation factors $\alpha>0, \tau \geq 0$. For $k=0,1,2, \ldots$ until the iteration sequence $\left\{\left(x^{(k)^{T}}, y^{(k)^{T}}\right)^{T}\right\}$ converges to the exact solution, compute the next iteration according to the following procedure

$$
\left\{\begin{array}{l}
(\widetilde{W}+\tau I) x^{(k+1)}=(1-\alpha) \widetilde{W} x^{(k)}+\tau x^{(k)}+\alpha \widetilde{T} y^{(k)}+\alpha p \\
\widetilde{W} y^{(k+1)}=(1-\alpha) \widetilde{W} y^{(k)}-\alpha \widetilde{T} x^{(k+1)}+\alpha q
\end{array}\right.
$$

Theorem 4.1. Let $W$ and $T \in \mathbb{R}^{n \times n}$ be symmetric positive definite and symmetric positive semidefinite, respectively. Also let $\tau$ be a nonnegative real constant, $\widetilde{W}=W+T, \widetilde{T}=T-W \in \mathbb{R}^{n \times n}$, 
and $\widetilde{S}=\widetilde{W}^{-1} \widetilde{T}$. Moreover, from Lemma 3.2 it can be seen that the eigenvalues of $\widetilde{S}$ are all real and nonnegative. Denote by

$$
\xi=\frac{x^{T} \widetilde{S} x}{x^{T} x}, \quad v=\frac{x^{T} \widetilde{W}^{-1} x}{x^{T} x} .
$$

Then the APGSOR method is convergent if and only if $\alpha$ satisfies

$$
0<\alpha<2
$$

or

$$
\left\{\begin{array}{l}
0<\alpha<\min \left\{2+\tau v_{\min }, \frac{2+\tau v_{\min }-\sqrt{\left(2+\tau v_{\min }\right)^{2}-4\left(1+\tau v_{\min }\right)\left(1-\tilde{\zeta}^{2}(\widetilde{S})\right)}}{1-\widetilde{\zeta}^{2}(\widetilde{S})}\right\}, \quad \text { if } 1-\widetilde{\zeta}^{2}(\widetilde{S}) \neq 0, \\
0<\alpha<\frac{2+2 \tau v_{\min }}{2+\tau v_{\min }}, \quad \text { if } 1-\widetilde{\zeta}^{2}(\widetilde{S})=0,
\end{array}\right.
$$

where $\xi_{\max }(\widetilde{S})$ is the largest eigenvalue in modulus of the matrix $\widetilde{S}=\widetilde{W}^{-1} \widetilde{T}$.

Proof. The proof is similar to that of Theorem 3.2. Hence it is omitted here.

\section{$5 \quad$ Numerical experiments}

In this section, we illustrate the feasibility and efficiency of the PGSOR and the APGSOR methods for solving complex symmetric system of linear equations. Meantime, we compare their numerical results including iteration steps (denoted by IT), elapsed CPU time in seconds (denoted by CPU) and relative residual error (denoted by RES) with those of the HSS, the MHSS and the GSOR methods. The numerical experiments are performed in MATLAB [version 7.14.0.739 (R2012a)] with machine precision $10^{-16}$ on a personal computer with 3.20 GHz 64-bit processor [Intel(R) Core(TM) i5-3470] and 8.00G memory.

In our implementations, the initial guess is chosen to be zero vector and the iteration is terminated once the relative residual error satisfies

$$
\text { RES : }=\frac{\left\|r^{(k)}\right\|_{2}}{\left\|r^{(0)}\right\|_{2}}<10^{-6}
$$

Example 5.1. Consider the complex symmetric linear system of the form (cf. $[10,16,19])$

$$
\left[\left(K+\frac{3-\sqrt{3}}{\tau} I\right)+i\left(K+\frac{3+\sqrt{3}}{\tau} I\right)\right] u=b,
$$

where $\tau$ is the time step-size, and $K=I_{m} \otimes V_{m}+V_{m} \otimes I_{m}$ with $V_{m}=h^{-2}$ tridiag $(-1,2,-1) \in$ $\mathbb{R}^{m \times m}$. $K$ is the five-point centered difference approximation of the negative Laplacian operator $L=-\triangle$ with homogeneous Dirichlet boundary conditions on uniform mesh in the unit square $[0,1] \times[0,1]$. Here $\otimes$ is the Kronecker product symbol and $h=1 /(m+1)$ is the discretization mesh-size. 
Table 1: Numerical results for Example 5.1.

\begin{tabular}{||lcccccc||}
\hline Method & & $16 \times 16$ & $32 \times 32$ & $64 \times 64$ & $128 \times 128$ & $256 \times 256$ \\
\hline HSS & IT & 44 & 65 & 97 & 136 & 191 \\
& CPU & 0.0579 & 0.3517 & 2.2193 & 17.5931 & 115.4806 \\
& RES & $9.16 \mathrm{e}-07$ & $9.82 \mathrm{e}-07$ & $9.84 \mathrm{e}-07$ & $9.26 \mathrm{e}-07$ & $9.51 \mathrm{e}-07$ \\
MHSS & IT & 40 & 54 & 73 & 98 & 133 \\
& CPU & 0.0231 & 0.1054 & 5.5891 & 5.2974 & 36.0572 \\
& RES & $9.67 \mathrm{e}-07$ & $9.61 \mathrm{e}-07$ & $9.41 \mathrm{e}-07$ & $9.35 \mathrm{e}-07$ & $9.99 \mathrm{e}-07$ \\
GSOR & IT & 19 & 22 & 24 & 26 & 26 \\
& CPU & 0.0189 & 0.0679 & 0.2319 & 1.3299 & 6.9214 \\
& RES & $9.02 \mathrm{e}-07$ & $9.24 \mathrm{e}-07$ & $6.38 \mathrm{e}-07$ & $4.82 \mathrm{e}-07$ & $9.46 \mathrm{e}-07$ \\
PGSOR & IT & 12 & 13 & 13 & 13 & 15 \\
& CPU & 0.0123 & 0.0356 & 0.1441 & 0.9854 & 1.1676 \\
& RES & $7.63 \mathrm{e}-07$ & $6.67 \mathrm{e}-07$ & $7.06 \mathrm{e}-07$ & $9.19 \mathrm{e}-07$ & $5.13 \mathrm{e}-07$ \\
APGSOR & IT & 5 & 5 & 5 & 5 & 5 \\
& CPU & 0.0046 & 0.0193 & 0.0817 & 0.3312 & 0.6756 \\
& RES & $4.04 \mathrm{e}-07$ & $5.79 \mathrm{e}-07$ & $5.22 \mathrm{e}-07$ & $9.14 \mathrm{e}-07$ & $2.06 \mathrm{e}-07$ \\
\hline
\end{tabular}

This complex symmetric linear system arises in centered difference discretization of $R_{22}$-Pade approximations in the time integration of parabolic partial differential equations [16]. In this example, $K$ is an $n \times n$ block diagonal matrix with $n=m^{2}$. In our tests, we take $\tau=h$. Furthermore, we normalize coefficient matrix and right-hand side of (5.1) by multiplying both by $h^{2}$. We take

$$
W=K+\frac{3-\sqrt{3}}{\tau} I \text { and } T=K+\frac{3+\sqrt{3}}{\tau} I .
$$

The right-hand vector $b$ is given with its $j$ th entry

$$
b_{j}=\frac{(1-i) j}{\tau(1+j)^{2}}, \quad j=1, \ldots, n .
$$

Example 5.2. Consider the complex symmetric linear system of the form (cf. $[5,10,19])$

$$
\left[\left(-\omega^{2} M+K\right)+i\left(\omega C_{V}+C_{H}\right)\right] u=b,
$$

where $M$ and $K$ are the inertia and the stiffness matrices, $C_{V}$ and $C_{H}$ are the viscous and hysteretic damping matrices, respectively. $\omega$ is the driving circular frequency and $K$ is defined the same as in Example 5.1.

This complex symmetric linear system arises in direct domain analysis of an $n$ degreeof-freedom (n-DOF) linear system [6]. In this example, $K$ is an $n \times n$ block diagonal matrix with $n=m^{2}$. We take $C_{H}=\mu K$ with $\mu$ being a damping coefficient, $M=I_{n}, C_{V}=10 I_{n}$. In 
Table 2: Numerical results for Example 5.2.

\begin{tabular}{||lcccccc||}
\hline Method & & $16 \times 16$ & $32 \times 32$ & $64 \times 64$ & $128 \times 128$ & $256 \times 256$ \\
HSS & IT & 86 & 153 & 284 & 540 & 1084 \\
& CPU & 0.1114 & 0.7887 & 6.3884 & 66.7546 & 672.4739 \\
& RES & $9.11 \mathrm{e}-07$ & $9.85 \mathrm{e}-07$ & $9.81 \mathrm{e}-07$ & $9.99 \mathrm{e}-07$ & $9.94 \mathrm{e}-07$ \\
MHSS & IT & 34 & 38 & 50 & 81 & 139 \\
& CPU & 0.0371 & 0.0889 & 0.4594 & 4.5696 & 38.9751 \\
& RES & $7.59 \mathrm{e}-07$ & $7.42 \mathrm{e}-07$ & $8.49 \mathrm{e}-07$ & $9.13 \mathrm{e}-07$ & $9.67 \mathrm{e}-07$ \\
GSOR & IT & 26 & 24 & 24 & 23 & 23 \\
& CPU & 0.0267 & 0.0526 & 0.2044 & 1.2558 & 8.1449 \\
& RES & $7.49 \mathrm{e}-07$ & $8.47 \mathrm{e}-07$ & $5.51 \mathrm{e}-07$ & $9.53 \mathrm{e}-07$ & $9.43 \mathrm{e}-07$ \\
PGSOR & IT & 12 & 12 & 12 & 16 & 20 \\
& CPU & 0.0118 & 0.0325 & 0.1337 & 1.2596 & 7.6351 \\
& RES & $9.78 \mathrm{e}-07$ & $4.21 \mathrm{e}-07$ & $9.66 \mathrm{e}-07$ & $9.22 \mathrm{e}-07$ & $9.97 \mathrm{e}-07$ \\
APGSOR & IT & 9 & 9 & 9 & 9 & 11 \\
& CPU & 0.0076 & 0.0292 & 0.1021 & 0.9953 & 4.5127 \\
& RES & $8.51 \mathrm{e}-07$ & $5.17 \mathrm{e}-07$ & $8.12 \mathrm{e}-07$ & $5.03 \mathrm{e}-07$ & $8.35 \mathrm{e}-07$ \\
\hline
\end{tabular}

Table 3: Choice of parameters: HSS, MHSS, GSOR vs PGSOR, APGSOR.

\begin{tabular}{||cccccccc||}
\hline \multirow{2}{*}{ Example } & \multirow{2}{*}{ Method } & & \multicolumn{7}{c||}{ Grid } \\
\cline { 3 - 8 } & & & $16 \times 16$ & $32 \times 32$ & $64 \times 64$ & $128 \times 128$ & $256 \times 256$ \\
\hline \multirow{6}{*}{ No.5.1 } & HSS & $\alpha_{\text {opt }}$ & 0.81 & 0.55 & 0.37 & 0.28 & 0.20 \\
& MHSS & $\alpha_{\text {opt }}$ & 1.06 & 0.75 & 0.54 & 0.40 & 0.30 \\
& GSOR & $\alpha_{\text {opt }}$ & 0.550 & 0.495 & 0.457 & 0.432 & 0.421 \\
& PGSOR & $\tau$ & 1.22 & 0.38 & 0.15 & 0.06 & 0.035 \\
& & $\alpha$ & 0.91 & 0.87 & 0.85 & 0.83 & 0.785 \\
& APGSOR & $\tau$ & 0.09 & 0.05 & 0.03 & 0.01 & 0.005 \\
& & $\alpha$ & 1.01 & 0.99 & 1.01 & 0.99 & 0.995 \\
& HSS & $\alpha_{\text {opt }}$ & 0.42 & 0.23 & 0.12 & 0.07 & 0.04 \\
& MHSS & $\alpha_{\text {opt }}$ & 0.21 & 0.08 & 0.04 & 0.02 & 0.01 \\
& GSOR & $\alpha_{\text {opt }}$ & 0.455 & 0.455 & 0.455 & 0.455 & 0.455 \\
& PGSOR & $\tau$ & 0.19 & 0.05 & 0.02 & 0.01 & 0.005 \\
& & $\alpha$ & 0.92 & 0.91 & 1.01 & 1.21 & 1.375 \\
& APGSOR & $\tau$ & 0.05 & 0.01 & 0.006 & 0.001 & 0.001 \\
& & $\alpha$ & 0.84 & 0.82 & 0.82 & 0.81 & 0.81 \\
\hline
\end{tabular}

addition, we set $\omega=\pi, \mu=0.02$ and the right-hand-side vector $b$ is chosen such that the exact solution of the linear system $(5.2)$ is $(1+i)(1,1, \ldots, 1)^{T} \in \mathbb{C}^{n}$. Similar to Example 5.1, the linear system is normalized by multiplying both sides with $h^{2}$. 


\section{Conclusion}

For a class of complex symmetric linear system we presented a parameterized generalized successive overrelaxation (PGSOR) method, which includes the generalized successive overrelaxation (GSOR) method as its special case. In addition, we demonstrate that our method is convergent under certain conditions. An accelerated PGSOR (APGSOR) method is established when $W$ is symmetric and positive definite and $T$ is symmetric and positive semi-definite. Observations of the numerical results let us know that the APGSOR is more effective than the PGSOR method. Again, numerical experiments show that our methods PGSOR and APGSOR are more efficient than the classical HSS, MHSS and GSOR methods comparing with iterative steps and CPU time required for solving the special complex symmetric system of linear equations in the work.

\section{Acknowledgments}

This project is supported partially by National Natural Science Foundation of China under Grant 11471150 and 11401281.

\section{References}

[1] S. R. Arridge, Optical tomography in medical imaging, Inverse Probl. 15 (1999), R41-R93.

[2] B. Poirier, Efficient preconditioning scheme for block partitioned matrices with structured sparsity, Numer. Linear Algebra Appl., 7 (2000), 715-726.

[3] L. C. Evans, Partial differential equations, American Mathematical Society, Providence, RI, 1998.

[4] A. Feriani, F. Perotti and V. Simoncini, Iterative system solvers for the frequency analysis of linear mechanical systems, Comput. Methods Appl. Mech. Engrg., 190 (2000), 1719-1739.

[5] D. Bertaccini, Efficient preconditioning for sequences of parametric complex symmetric linear systems, Electr. Trans. Numer. Anal., 18 (2004), 49-64.

[6] M. Benzi and D. Bertaccini, Block preconditioning of real-valued iterative algorithms for complex linear systems, IMA J. Numer. Anal., 28 (2008), 598-618.

[7] A. Bunse-Gerstner and R. Stöver, On a conjugate gradient-type method for solving complex symmetric linear systems , Linear Algebra Appl., 287 (1999), 105-123.

[8] Y. Saad, Iterative methods for sparse linear systems, Second Edition, Philadelphia, PA, 2003.

[9] Z.-Z. Bai, G. H. Golub and M. K. Ng, Hermitian and skew-Hermitian splitting methods for non-Hermitian positive definite linear systems, SIAM J. Matrix Anal. Appl., 24 (2003), 603626.

[10] Z.-Z. Bai, M. Benzi, F. Chen, Modified HSS iteration methods for a class of complex symmetric linear systems, Computing, 87 (2010), 93-111.

[11] Z.-Z. Bai, M. Benzi, F. Chen and Z.-Q. Wang, Preconditioned MHSS iteration methods for a class of block two-by-two linear systems with applications to distributed control problems, IMA J. Numer. Anal., 33 (2013), 343-369.

[12] Z.-Z. Bai, Rotated block triangular preconditioning based on PMHSS, Sci. China Math., 56 (2013), 2523-2538. 
[13] Z.-Z. Bai, M. Benzi and F. Chen, On preconditioned MHSS iteration methods for complex symmetric linear systems, Numer. Algorithms, 56 (2011), 297-317.

[14] X. Li, A.-L. Yang and Y.-J. Wu, Lopsided PMHSS iteration method for a class of complex symmetric linear systems, Numer. Algorithms, 66 (2014), 555-568.

[15] Y.-J. Wu, X. Li and J.-Y. Yuan, A non-alternating preconditioned HSS iteration method for non-Hermitian positive definite linear systems, Comput. Appl. Math., 36 (2017), 367-381.

[16] O. Axelsson and A. Kucherov, Real valued iterative methods for solving complex symmetric linear systems, Numer. Linear Algebra Appl., 7 (2000), 197-218.

[17] O. Axelsson, M. Neytcheva and B. Ahmad, A comparison of iterative methods to solve complex valued linear algebraic systems, Numer. Algorithms, 66 (2014), 811-841.

[18] Z.-Z. Bai, On preconditioned iteration methods for complex linear systems, J. Engrg. Math., 93 (2015), 41-60.

[19] X.-A. Li, W.-H. Zhang and Y.-J. Wu, On symmetric block triangular splitting iteration method for a class of complex symmetric system of linear equations, Appl. Math. Lett., 79 (2018), 131-137.

[20] D. K. Salkuyeh, D. Hezari and V. Edalatpour, Generalized successive overrelaxation iterative method for a class of complex symmetric linear system of equations, Int. J. Comput. Math., 92 (2015), 802-815.

[21] D. Hezari, V. Edalatpour and D. K. Salkuyeh, Preconditioned GSOR iterative method for a class of complex symmetric system of linear equations, Numer. Linear Algebra Appl., 22 (2015), 761-776.

[22] D. Hezari, D. K. Salkuyeh and V. Edalatpour, A new iterative method for solving a class of complex symmetric system of linear equations, Numer. Algorithms, 73 (2016), 927-955.

[23] Z.-Z. Liang and G.-F. Zhang, On SSOR iteration method for a class of block two-by-two linear systems, Numer. Algorithms, 71 (2016), 655-671.

[24] O. Axelsson, Iterative Solution Methods, Cambridge University Press, Cambridge, 1994. 